\title{
RELAÇÃO ENTRE ATIVIDADE FÍSICA E SÍNDROME DE BURNOUT EM ESTUDANTES UNIVERSITÁRIOS: REVISÃO SISTEMÁTICA
}

\author{
Gelcemar Oliveira Farias \\ Universidade do Estado de Santa Catarina, Florianópolis, Santa Catarina, Brasil. \\ Iris Dantas da Mota \\ Universidade do Estado de Santa Catarina, Florianópolis, Santa Catarina, Brasil. \\ Ana Paula Ramos Marinho \\ Centro Universitário Facvest, Lages, Santa Catarina, Brasil. \\ Jorge Both \\ Universidade Estadual de Londrina, Londrina, Paraná, Brasil. \\ Monica Bredun da Veiga \\ Universidade do Estado de Santa Catarina, Florianópolis, Santa Catarina, Brasil.
}

\begin{abstract}
Resumo
Esta revisão sistemática visa analisar a literatura científica sobre a relação entre atividade física e a síndrome de Burnout em estudantes universitários. Esta revisão seguiu as recomendações do Preferred reporting items for Systematic Review and Meta-análise (PRISMA). A análise foi feita por meio da revisão sistemática de artigos publicados nas bases de dados $P u b$ Med, SCOPUS, Web of Science e PsycInfo, em português, inglês e espanhol. Foram identificados 99 estudos, todos publicados em inglês, porém apenas três estudos preencheram os critérios de inclusão. Os sintomas da síndrome de Burnout estiveram presentes em uma quantidade significativa da amostra. O nível de atividade física influenciou a percepção dos sintomas, tendo os alunos mais ativos apresentado escores menores.
\end{abstract}

Palavras-chave: Esgotamento Profissional. Atividade Física. Universidades. Estudantes.

\section{RELATIONSHIP BETWEEN PHYSICAL ACTIVITY AND BURNOUT SYNDROME IN UNIVERSITY STUDENTS: SYSTEMATIC REVIEW}

\begin{abstract}
This systematic review aims to analyze the scientific literature on the relationship between physical activity and Burnout syndrome in university students. This review followed the recommendations of the Preferred reporting items for Systematic Review sand meta-analysis (PRISMA). The analysis was done through a systematic review of articles published in the PubMed, SCOPUS, Web of Science and PsycInfo databases in Portuguese, English and Spanish. We identified 99 studies, all published in English, but only three studies met the inclusion criteria. The symptoms of Burnout syndrome were present in a significant amount of the sample. The level of physical activity influenced the perception of symptoms, with the most active students showing lower scores.
\end{abstract}

Keywords: Burnout. Professional. Exercise. Universities. Students. 


\title{
RELACIÓN ENTRE ACTIVIDAD FÍSICA Y SÍNDOME DE BURNOUT EN ESTU- DIANTES UNIVERSITARIOS: REVISIÓN SISTEMÁTICA
}

\begin{abstract}
Resumen
Esta revisión sistemática busca analizar la literatura científica sobre la relación entre la actividad física y el síndrome de Burnout en estudiantes universitarios. Esta revisión siguió las recomendaciones de los elementos de información de preferencia para el análisis de análisis de análisis (PRISMA). El análisis se realiza a través de la revisión sistemática de los artículos publicados en las bases de datos PubMed, Scopus, Web of Science y PsycInfo en Portugués, Inglés y Español. Se identificaron 99 estudios, todos publicados en inglés, pero sólo tres estudios cumplieron los criterios de inclusión. Los síntomas del síndrome de Burnout estuvieron presentes en una cantidad significativa de la muestra. El nivel de actividad física influenció la percepción de los síntomas, teniendo los alumnos más activos demostrados escores menores.

Palabras clave: Agotamiento Profesional. Actividad física. Universidades. Estudiantes.
\end{abstract}

\section{Introdução}

O contexto universitário é pautado por desafios e expectativas relacionados ao ambiente educacional, vivenciados pelos estudantes no decorrer de sua formação inicial, quando buscam a qualificação que será determinante para sua carreira. Inicialmente, o período de transição para o ensino superior é vivenciado com sentimentos de ansiedade frente às novidades e às exigências institucionais e acadêmicas, às quais os alunos precisam se adaptar, de modo a gerar mecanismos de repostas que lhes garantam a permanência e o sucesso no ensino superior e na pós-graduação (ALMEIDA, 2007; FOGAÇA et al., 2016; MESSAS et al., 2015).

No decorrer da formação inicial, os mecanismos individuais desenvolvidos pelos alunos para enfrentar as exigências e as demandas de estudo podem ocasionar o surgimento dos sintomas da síndrome de Burnout em estudantes que se encontram em diferentes etapas da formação, levando instituição e acadêmicos a criarem estratégias para o enfrentamento de tal situação (SZILVIA et al., 2014).

Estudos nacionais e internacionais que abordam a síndrome de Burnout em estudantes reportam tanto investigações no contexto do ensino fundamental e do ensino médio (ARINO; HERNADEZ, 2016), na formação do ensino superior, nos mais variados cursos de formação inicial (BACKOVIĆ et al., 2012; DYRBYE; SCHANAFELT, 2016), como investigações na pós-graduação (GALDINO et al., 2016; SOUZA et al., 2010), o que revela determinantes que se tornam relevantes para a futura intervenção profissional. Na formação inicial, as demandas resultantes do ritmo de vida frenético dos estudantes, as solicitações acadêmicas e a baixa incidência de atividade física dessa população podem ser alguns dos indicadores da síndrome de Burnout.

A síndrome de Burnout caracteriza-se como um estado de elevada exaustão emocional, despersonalização e redução do sentimento de realização pessoal (MASLACH; JACKSON, 1981). Os estudantes acometidos tendem a se sentir emocionalmente esgotados e incapazes de realizar as tarefas acadêmicas, de tal forma que desenvolvem atitudes cínicas e de desvalorização do próprio desempenho acadêmico, culminando, em alguns casos, no abandono do curso e no comprometimento negativo de sua saúde física e psicológica (CAMPBELL et al., 2010).

Assim, é importante que as instituições de ensino superior desenvolvam, junto com a comunidade acadêmica, estratégias de prevenção ou de coping da síndrome de Burnout que amenizem os desconfortos ocasionados por seus sintomas. Nesse cenário, a atividade física 
pode ser elencada como uma maneira eficiente de prevenir e amenizar os sintomas da síndrome de Burnout na dimensão individual, contribuindo para melhorar o desempenho das funções acadêmicas dos estudantes. Estudos revelaram os benefícios da atividade física tanto sobre a saúde psicológica como nas esferas biológica e social, o que demonstra que pessoas mais ativas fisicamente encontravam-se mais protegidas de desordens na saúde (BATISTA; ORNELLAS, 2013; GUEDES; GUEDES, 1995; LEITÃO et al., 2000; MANOSSO et al., 2014; MATSUDO; MATSUDO, 2000; MELLO et al., 2005; SCHENKEL et al., 2011).

Divulgar os resultados de pesquisas envolvendo esta temática no ambiente acadêmico, além da implementação de práticas corporais pelas instituições de ensino, assume grande relevância por contribuir para a promoção da saúde dos estudantes universitários e motivá-los a adotarem um estilo de vida saudável. A prática de atividades físicas e esportivas reflete na melhor qualidade tanto dos estudos como das condições de aprendizagem dos acadêmicos (BIELEMANN et al., 2007).

Apesar da importância social e acadêmica relacionada à temática em questão, pouco tem sido investigado no que tange à relação entre atividade física e síndrome de Burnout. A maioria dos estudos limita-se a identificar fatores individuais da síndrome em estudantes universitários sem abordar os fatores de prevenção e de tratamento (CARLOTTO; CÂMARA, 2008; CAMPOS et al., 2012; GUIMARÃES, 2014; TOMASCHEWSKI-BARLEM et al., 2013). Nesse sentido, o objetivo do estudo foi realizar uma revisão sistemática da produção científica sobre as relações entre síndrome de Burnout e atividade física em estudantes inseridos em cursos de graduação e de pós-graduação.

\section{Método}

A revisão sistemática foi registrada no International Prospective Register of Systematic Reviews (PROSPERO), sob o número CRD42016050752. O delineamento seguiu as recomendações indicadas no modelo Preferred Reporting Items for Systematic Review and Meta-Analyses (PRISMA), que define critérios específicos para esse tipo de estudo (MOHER et al., 2009).

Para a seleção automática e manual dos artigos foram definidos os seguintes critérios de inclusão: (a) estudos publicados nos idiomas inglês, espanhol e português; (b) estudos que investigaram a relação entre síndrome de Burnout e atividade física (incluindo exercício físico); (c) estudos que tivessem como amostra estudantes universitários de graduação e pósgraduação; (d) estudos disponíveis em acesso completo e digital; (e) trabalhos publicados até novembro de 2016. Do mesmo modo, os critérios de exclusão foram assim definidos: estudos que se caracterizavam metodologicamente como revisão sistemática, estudos de validação de questionários, estudos pilotos, protocolos de estudo, dissertações, teses, capítulos de livros, suplementos ou comentários dos editores.

A busca automática das informações foi realizada nas bases de dados PubMed, SCOPUS (Elsevier), Web of Science e PsycInfo, enquanto a busca manual ocorreu nas listas de referência dos artigos selecionados. Esse processo de recolha das informações foi realizado no mês de novembro de 2016, por dois pesquisadores, de modo a evitar erros no processo inicial de coleta dos dados. Os mesmos descritores foram utilizados em todas as bases com as adaptações necessárias para cada uma. A equação de pesquisa utilizada foi: Burnout AND motor activity, OR physical activity, OR exercise, AND students, AND Maslach Burnout Inventory, OR MBI.

Após a coleta de dados foram identificados os títulos e resumos dos artigos, encontrados em cada base de indexação. Além dessa ação, foram realizadas buscas nas referências bibliográficas dos artigos, para identificar outros estudos que pudessem contribuir com a análise atual. Após a leitura dos títulos e resumos, foram aplicados os critérios de inclusão e de 
exclusão dos artigos pelos pesquisadores que sistematizaram a coleta de dados, de modo a incluir ou excluir aqueles que não se adequavam à temática investigada, bem como os duplicados. Nesse momento, houve a necessidade de um terceiro pesquisador para a realização do consenso, ou seja, a inclusão ou a exclusão de artigos nos quais os pesquisadores apresentaram dúvidas ou discordância. Mediante a efetivação do consenso, foi realizada a leitura na íntegra e o fichamento de todos os artigos selecionados.

Para a avaliação da validade externa e interna e o poder estatístico dos artigos foi utilizada a adaptação da escala de Downs e Black (1998), presente no estudo de Boing et al. (2016), composta por 19 questões que pontuavam 20 pontos no total, pois se atribuía pontuação 1 quando a resposta era pertinente, exceto para a questão 5, que possuía pontuação 2. Para tanto, os textos selecionados foram analisados considerando o objetivo (foco de investigação), o local de realização do estudo, os procedimentos metodológicos (tipo de estudo, amostra e instrumentos de coleta de dados) e os resultados.

\section{Resultados}

Após a busca inicial nas bases de dados, foi encontrado um total de 99 artigos que correspondiam aos descritores e aos critérios estabelecidos na metodologia. Dentre os trabalhos encontrados, 14 estavam litados no PubMed, 70 na base SCOPUS (Elsevier), sete na base Web of Science, e oito na base PsycInfo. Foram excluídos, inicialmente, oito estudos por referências cruzadas, restando 91 artigos. Destes, 73 trabalhos foram excluídos após a leitura dos títulos por estar fora da temática estabelecida, restando 18 artigos para a fase da leitura dos resumos.

Após a leitura dos resumos dos 18 artigos selecionados, 12 foram excluídos por não contemplarem a temática da pesquisa. Restaram apenas seis estudos para a leitura integral, dos quais somente três artigos atendiam aos critérios de inclusão estabelecidos. No entanto, um desses estudos foi excluído por estar em idioma diferente do inglês, do espanhol e do português. Posteriormente, foi realizada busca através das referências bibliográficas dos artigos selecionados para o estudo atual, sendo incluído mais um estudo por contemplar a temática e os objetivos desta pesquisa. Obtiveram-se, portanto, ao final da seleção, três estudos adequados aos critérios de inclusão, que foram inseridos para contemplar este estudo. A Figura 1 apresenta o fluxograma da seleção de estratégia dos estudos de acordo com as diretrizes do PRISMA.

Figura 1: Fluxograma de estratégia de seleção dos estudos de acordo com as diretrizes PRISMA (2009).
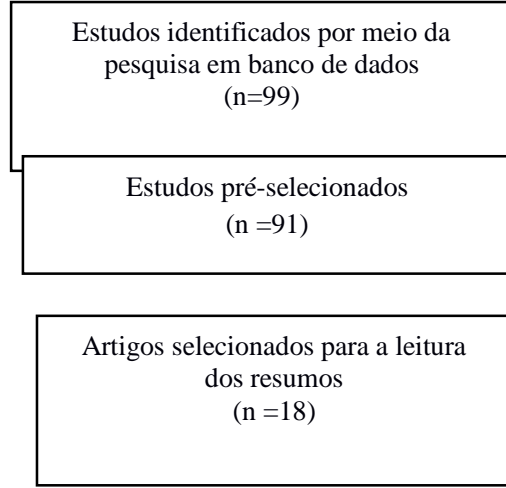

Estudos incluídos para a leitura integral

$(\mathrm{n}=6)$

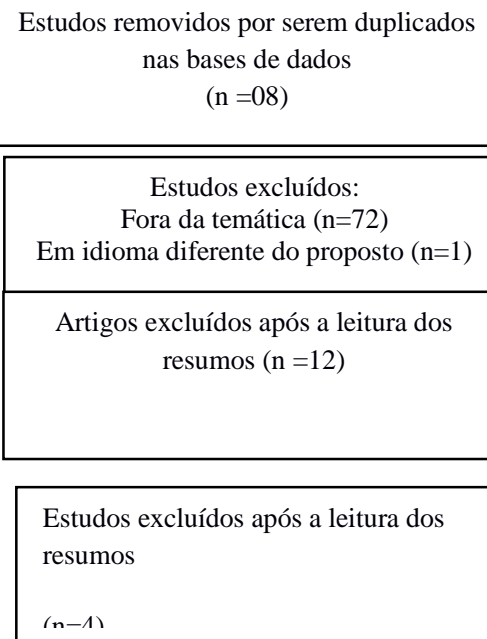

Estudos removidos por serem duplicados nas bases de dados $(\mathrm{n}=08)$

Estudos excluídos:

Fora da temática $(n=72)$

Em idioma diferente do proposto $(n=1)$

Artigos excluídos após a leitura dos resumos $(\mathrm{n}=12)$

Estudos excluídos após a leitura dos resumos

$(n-4$ 
Estudos incluídos para a revisão sistemática selecionados das bases $(n=2)$

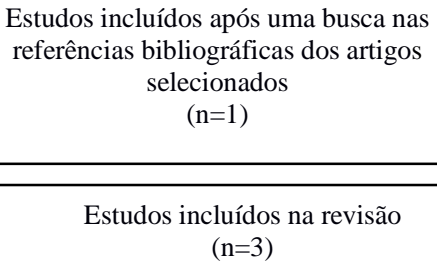

Estudos incluídos após uma busca nas referências bibliográficas dos artigos selecionados $(\mathrm{n}=1)$

Estudos incluídos na revisão $(n=3)$

Fonte: Os autores, 2017.

A apreciação preliminar das três produções científicas (WEIGHT et al., 2013; CECIL et al., 2014; FARES et al., 2015) selecionadas revelou a atualidade da temática, visto que todos os estudos são internacionais e foram publicados nos anos 2013, 2014 e 2015, totalizando uma publicação por ano. A amostra total dos estudos foi de 1149 estudantes, todos do curso de medicina, sendo 580 homens e 569 mulheres, representando aproximadamente $50,5 \%$ e $49,5 \%$, respectivamente. Destaca-se que as investigações foram realizadas em diferentes países: Estados Unidos, Reino Unido e Líbano. No Quadro 1 são apresentados os principais objetivos dos estudos.

Quadro 1- Autoria e objetivos dos estudos incluídos.

\begin{tabular}{|c|l|}
\hline Autores & \multicolumn{1}{c|}{ Objetivo } \\
\hline $\begin{array}{c}\text { Weight et al. } \\
\text { (2013) }\end{array}$ & $\begin{array}{l}\text { Analisar os efeitos de um programa de atividade física sobre qualidade } \\
\text { de vida e Burnout entre residentes médicos e residentes médicos bolsis- } \\
\text { tas de um grande centro acadêmico. }\end{array}$ \\
\hline Cecil et al. (2014) & $\begin{array}{l}\text { Investigar, em estudantes do curso de graduação em medicina de duas } \\
\text { universidades, a prevalência de Burnout e sua associação com os com- } \\
\text { ponentes de estilo de vida e saúde (atividade física, consumo de álcool, } \\
\text { dieta, tabagismo), idade, sexo, ano de estudo e instituição frequentada. }\end{array}$ \\
\hline $\begin{array}{c}\text { Fares et al. } \\
\text { (2015) }\end{array}$ & $\begin{array}{l}\text { Avaliar a ocorrência de estresse e Burnout nos estudantes de medicina e } \\
\text { sua associação com as atividades extracurriculares (atividades físicas, } \\
\text { sociais, de música, de leitura). }\end{array}$ \\
\hline
\end{tabular}

Fonte: Os autores, 2017.

Observa-se que em todos os estudos (Quadro 1) a prática da atividade física foi relacionada com outras variáveis, como: sexo, idade, atividade física, consumo de álcool, dieta, tabagismo, ano de estudo e atividades extracurriculares (música, leitura e atividades sociais).

O estudo de Weight etal. (2013) investigou os benefícios da atividade física em relação à qualidade de vida e ao Burnout por meio de um programa de intervenção com duração de 12 semanas, que envolveu exercícios eletivos, baseados em equipe, incluindo exercícios aeróbicos de alta performance (alta e moderada intensidade) e exercícios de alongamento, como complemento do estudo feito entre residentes médicos e residentes médicos bolsistas de um centro acadêmico. Cecil et al. (2014) averiguaram a prevalência de Burnout e sua associação com os componentes de estilo de vida e saúde (atividade física, consumo de álcool, dieta, tabagismo) e com as variáveis: idade, sexo, ano de estudo e instituição frequentada pelos estudantes do curso de graduação em medicina de duas universidades. Fares et al. (2015) analisaram a ocorrência de estresse e Burnout nos estudantes de medicina, bem como sua associação com atividades extracurriculares (atividade física, música, leitura e atividades sociais).

A coleta de dados dos estudos foi realizada por meio de distintos instrumentos: Questionário Internacional de Atividade Física (IPAQ), utilizado em um dos estudos (CECIL et al., 
2014); questionário sobre hábitos e frequência com que praticam atividade física (exercise habits) (WEIGHT et al., 2013); e questionário sobre saúde em geral (GHQ-12) (FARES et al., 2015). Além dos questionários simples de frequência, Fares et al. (2015) realizaram avaliações relativas à alimentação e ao consumo de álcool, através dos questionários Alcohol Use Questionnaire (AUQ) e Food Frequency Questionnaire (FFQ). O Maslach Burnout Inventory foi encontrado em todos os estudos, por ser a ferramenta considerada padrão para diagnóstico da síndrome de Burnout (WEIGHT et al., 2013; CECIL et al., 2014; FARES et al., 2015). No que se refere aos tipos de estudo, observou-se que as três investigações foram delineadas por meio de abordagem quantitativa dos dados.

Com relação às pontuações obtidas por meio da Escala Metodológica (DOWNS; BLACK, 1998), verificou-se que no estudo que realizou intervenção o escore foi de 16 pontos, estando as lacunas observadas relacionadas às características de comunicação; à validade externa e interna (viés); à não descrição das características dos indivíduos que desistiram do estudo no decorrer da pesquisa; à falta de definição do embasamento dos resultados (não detalhamento dos principais fatores de confusão); aos aspectos referentes à validade interna dos estudos. Quanto aos estudos que não realizaram intervenção, os escores foram de 13 e 18 pontos, com média de 15,5 pontos. A avaliação metodológica evidenciou que nenhum artigo atingiu valores máximos, deixando nítidas algumas falhas metodológicas na execução dessas pesquisas. No entanto, nenhum dos artigos ficou com valores abaixo de 11, o que pode caracterizar a amostra como 'boa', de acordo com a classificação qualitativa da Downs e Black (1998).

Destaca-se que a análise de estudos, de acordo com procedimentos metodológicos e resultados, está apresentada no Quadro 2.

Quadro 2: Análise do método, tipo de estudo, amostragem, instrumentos e resultados.

\begin{tabular}{|c|c|c|c|c|c|c|}
\hline Referência & Local & $\begin{array}{c}\text { Tipo de estu- } \\
\text { do }\end{array}$ & Amostra & Instrumento & Resultados & Escore* \\
\hline $\begin{array}{l}\text { Weight et } \\
\text { al. (2013) }\end{array}$ & $\begin{array}{l}\text { Estados } \\
\text { Unidos }\end{array}$ & Experimental & $\begin{array}{c}628 \\
\text { (368 ho- } \\
\text { mens, } 260 \\
\text { mulheres) }\end{array}$ & $\begin{array}{l}\text { Body mass in- } \\
\text { dex (BMI), exer- } \\
\text { cise habits, QOL } \\
\text { (Quality of life), } \\
\text { Maslach Burn- } \\
\text { out Inventory } \\
\text { (MBI), and pro- } \\
\text { gram participa- } \\
\text { tion status. }\end{array}$ & $\begin{array}{l}\text { Os participantes do } \\
\text { programa de ativi- } \\
\text { dade física percebe- } \\
\text { ram qualidade de } \\
\text { vida maior em rela- } \\
\text { ção aos não partici- } \\
\text { pantes. Os sintomas } \\
\text { da síndrome de Bur- } \\
\text { nout (despersonali- } \\
\text { zação, exaustão } \\
\text { emocional e outros } \\
\text { sintomas) foram } \\
\text { menores nos partici- } \\
\text { pantes (5\%), apesar } \\
\text { da diferença estatís- } \\
\text { tica não ser significa- } \\
\text { tiva. }\end{array}$ & $16^{* *}$ \\
\hline $\begin{array}{c}\text { Cecil et al. } \\
(2014)\end{array}$ & $\begin{array}{c}\text { Reino } \\
\text { Unido e } \\
\text { Escócia }\end{array}$ & $\begin{array}{c}\text { Estudo trans- } \\
\text { versal }\end{array}$ & $\begin{array}{c}356 \\
(124 \text { ho- } \\
\text { mens, } 232 \\
\text { mulheres) }\end{array}$ & $\begin{array}{c}\text { Maslach Burn- } \\
\text { out Inventory } \\
\text { (MBI); alcohol } \\
\text { Use (AUQ); } \\
\text { Questionnaire; } \\
\text { International } \\
\text { physical Activity } \\
\text { Questionnaire } \\
\text { (IPAQ); Food }\end{array}$ & $\begin{array}{c}\text { A atividade física } \\
\text { influenciou, signifi- } \\
\text { cativamente e de } \\
\text { forma positiva, os } \\
\text { níveis de exaustão } \\
\text { emocional e realiza- } \\
\text { ção pessoal. }\end{array}$ & 13\# \\
\hline
\end{tabular}




\begin{tabular}{|c|c|c|c|c|c|c|}
\hline & & & $\begin{array}{c}\text { frequency } \\
\text { Questionnaire } \\
\text { (FFQ). }\end{array}$ & & \\
\hline $\begin{array}{c}\text { Fares et al. } \\
\text { (2015) }\end{array}$ & Líbano & $\begin{array}{c}\text { Estudo trans- } \\
\text { versal }\end{array}$ & $\begin{array}{c}165 \\
(88 \text { ho- } \\
\text { mens, } 77 \\
\text { mulheres) }\end{array}$ & $\begin{array}{c}\text { 12-item General } \\
\text { Health Ques- } \\
\text { tionnaire (GHQ- } \\
\text { 12), and the } \\
\text { Maslach Burn- } \\
\text { out Inventory- } \\
\text { Student Survey } \\
\text { (MBI-SS) }\end{array}$ & $\begin{array}{c}\text { O exercício físico foi } \\
\text { significativamente } \\
\text { associado ao baixo } \\
\text { estresse. A análise } \\
\text { mostrou que indiví- } \\
\text { duos do sexo femi- } \\
\text { nino sofrem de alta } \\
\text { exaustão emocional, } \\
\text { síndrome de Bur- } \\
\text { nout e alto estresse, } \\
\text { assim como estudan- } \\
\text { tes do primeiro ano } \\
\text { do curso de medici- } \\
\text { na. }\end{array}$ & $18 \#$ \\
\hline
\end{tabular}

Legenda: *Escore da escala de avaliação metodológica Downs e Black (1998)*.**Pontuação escala Downs e Black (1998), original \#Pontuação escala Downs e Black (1998), adaptada. Fonte: Os autores, 2017.

Os estudos demonstraram que sintomas da síndrome de Burnout estão presentes de forma significativa na amostra, com escores expressivos nas três dimensões da síndrome. Nível de atividade física, sexo, ano de estudo e estilo de vida também influenciaram a percepção dos sintomas da síndrome e a qualidade de vida. Os alunos mais ativos demonstraram escores menores nas dimensões da síndrome, bem como uma percepção de qualidade de vida mais elevada (CECIL et al., 2014; FARES et al., 2015; WEIGHT et al., 2013).

No estudo de Weight et al. (2013) foram constatados níveis elevados na dimensão exaustão emocional e despersonalização dos estudantes de medicina. Após um período de inserção em um programa de atividade física de 12 semanas, a percepção da qualidade de vida aumentou entre os acadêmicos e a percepção dos sintomas da síndrome diminuiu, embora estatisticamente a diferença não tenha sido considerada significativa. Fares et al. (2015) constataram que grande parte dos alunos estavam estressados e acometidos pela Burnout e verificaram que a prática de atividade física como componente extracurricular estava significativamente associada ao baixo estresse.

Cecil et al. (2014) investigaram a prevalência de Burnout entre os estudantes de medicina e a associação entre Burnout e comportamentos relacionados à saúde, incluindo atividade física regular. Os resultados demonstraram que a síndrome está presente em significativa quantidade de estudantes, sendo o escore de exaustão emocional o mais elevado, seguido de baixa realização pessoal e despersonalização. O estudo diagnosticou que os comportamentos de saúde podem ser preditores para o desenvolvimento da síndrome. A atividade física foi a mais preditiva de todas as variáveis de comportamento, com altos índices de realização pessoal e escores menores em exaustão emocional.

\section{Discussão}

As investigações sobre a síndrome de Burnout e sua relação com o nível de atividade física em estudantes universitários vem despertando o interesse da comunidade científica ao longo dos anos. Contudo, ainda são incipientes as investigações que reportam a temática. Torna-se, portanto, necessário o engajamento da comunidade científica para aprofundar tais conhecimentos, tendo em vista a demanda de três publicações que compuseram esta revisão 
sistemática. Ao dissociar a síndrome de Burnout do nível de atividade física em estudantes universitários são perceptíveis investigações sobre a temática em distintos países, tais como Brasil (GARBIN et al., 2012; SANTOS et al., 2017), Portugal (SILVA; SIMÕES; VAZ, 2015), Chile (GÓMEZ et al., 2015) e Cuba (ROSALES, 2012).

A síndrome de Burnout em estudantes universitários foi identificada principalmente nos cursos relacionados à área da saúde, como enfermagem (TOMASCHEWSKI-BARLEM et al., 2013), medicina (MORI; VALENTE; NASCIMENTO, 2012), fisioterapia (SCHENKEL et al., 2012) e psicologia (CARLOTTO et al., 2010). Os estudos revelaram a presença dos sintomas da síndrome em acadêmicos de diferentes cursos de graduação, evidenciando que, no decorrer da preparação para o exercício profissional, os estudantes, em diferentes etapas da formação inicial, apresentaram sinais da síndrome de Burnout.

De maneira independente, a atividade física também foi investigada nos estudantes universitários (MARCONDELLI; COSTA; SCHMITZ, 2008; FONTES; VIANNA, 2009; RODRIGUES; CHEIK; MAYER, 2008; SILVA et al.,2007). Os estudos demonstraram a prevalência da síndrome nos estudantes sedentários, com valores significativos em relação aos que foram considerados ativos. Entre os motivos para explicar esse comportamento, foram elencados: falta de tempo e de disposição para a prática de atividades físicas; insuficiência de condições financeiras; ausência de local adequado para praticar atividades físicas ao ar livre.

Corroborando os estudos apresentados anteriormente, as investigações que fizeram parte desta revisão (CECIL et al., 2014; FARES et al., 2015; WEIGHT et al., 2013) identificaram a presença dos sintomas da síndrome de Burnout em estudantes de medicina. Em todos os estudos, a prática da atividade física influenciou positivamente na redução da exaustão emocional e da despersonalização e no aumento da realização pessoal. Os universitários mais ativos apresentaram menores índices nas dimensões da síndrome.

Embora os estudos em tela tenham revelado relação inversa entre o nível de atividade física e a menor disposição ao desenvolvimento da síndrome de Burnout, ressalta-se a importância de fomentar novas investigações em estudantes universitários, com o intuito de prevenir, neutralizar ou cessar as manifestações de Burnout em sua fase mais incipiente, de forma que as tarefas e as demandas de pesquisas sejam exercidas com êxito e entusiasmo no decorrer de todo o processo de formação inicial.

Com relação ao método de estudo, foi utilizado prioritariamente o instrumento de coleta de dados denominado Maslach Burnout Inventory - Student Survey (MBI-SS) em todas as investigações encontradas, para mensurar as dimensões da síndrome, por ser reconhecido como ferramenta padrão em pesquisas na área. Nas investigações com estudantes universitários, o instrumento segue, em relação às dimensões, a mesma matriz estabelecida no instrumento primário por Maslach e Jackson (1981).

A exaustão emocional é caracterizada por um sentimento muito forte de tensão emocional que produz sensação de esgotamento, de falta de energia e de recursos emocionais para lidar com as rotinas da prática profissional. A despersonalização é resultado do desenvolvimento de sentimentos e atitudes negativas, por vezes, indiferentes e cínicas. A realização profissional identifica-se pela tendência de estar insatisfeito com as condições que afetam as habilidades interpessoais relacionadas com a prática profissional (MASLACH; JACKSON, 1981).

$\mathrm{Na}$ avaliação em estudantes universitários tais dimensões referem-se a seus sentimentos e a suas emoções durante o curso: exaustão emocional, caracterizada pela sensação de estar exausto em virtude das exigências do estudo; descrença, entendida como o desenvolvimento de uma atitude cínica e distanciada com relação ao estudo; eficácia profissional, caracterizada pela percepção de estarem sendo incompetentes como estudantes (BACKOVIĆ et al., 2012; CARLOTTO; NAKAMURA; CÂMARA, 2006; ROSALLES, 2013; CABALLERO; BRESO; GONZALEZ, 2015; DYRBYE; SHANAFELT, 2016). 
Alguns dos sintomas típicos da síndrome são relatados por Benevides-Pereira (2002) como sintomatologias que afetam o indivíduo nas esferas físicas, psíquicas, comportamentais e defensivas. Entre elas se destacam: fadiga constante e progressiva, distúrbios do sono, dores musculares, irritabilidade, perda de iniciativa, incapacidade para relaxar, falta de atenção e de concentração, alterações da memória, impaciência, tendência ao isolamento, absenteísmo e perda de interesse pelo trabalho.

A literatura mostra também estudos que englobam a prática de atividade física com ação benéfica sobre parte desses sintomas, seja como prevenção, seja como tratamento (DIAS, 2014; FLESHNER, 2005; FREITAS et al., 2014; MEJÍA, 2011; PÉREZ et al., 2014; RONCADA; SALAZAR, 2010; SILVA et al., 2014;). Os desfechos de algumas pesquisas confirmam as evidências de que a atividade física tem implicações positivas para a saúde.

A prática da atividade física nas rotinas diárias relacionada a um estilo de vida saudável pode ser considerada uma estratégia de enfrentamento dos fatores desencadeantes da síndrome de Burnout por contribuir para a melhora da saúde, da qualidade de vida e do desempenho das funções ocupacionais dos indivíduos (MATSUDO; MATSUDO, 2000; MANOSSO et al., 2014). O estilo de vida saudável está vinculado a fatores relacionados à saúde geral e associados ao aumento do bem-estar psicológico e à diminuição do desenvolvimento de diversas doenças. Tal estilo inclui nutrição balanceada, controle do estresse, adoção de comportamentos preventivos, prática de atividades físicas (NAHAS; BARROS; FRANCALACCI, 2000).

Ainda são poucos os estudos científicos publicados que abordam a temática que envolve a relação entre atividade física e Burnout em estudantes universitários. No entanto, pelos estudos selecionados nesta revisão sistemática, percebeu-se implicação positiva entre as variáveis, considerando a melhora na percepção da qualidade de vida do estudante. Investigar a atividade física e a síndrome de Burnout torna-se significativa, por permitir a reflexão e ajudar o incremento da qualidade do ensino. Tais investigações são importantes para auxiliar na construção de matrizes curriculares e na elaboração e efetivação de projetos pedagógicos que visem minimizar os desconfortos ocasionados no período da formação inicial e que busquem a obtenção de melhor qualidade de vida e melhor rendimento acadêmico.

No cenário universitário, as demandas formativas atuais talvez sejam mecanismos que favoreçam a síndrome de Burnout nos estudantes, no entanto, raras são as investigações que reportam essa ação. Na área da Educação Física, percebe-se uma lacuna nas investigações que envolvem esta temática, evidenciando a necessidade de ampliação das investigações, de modo a contribuir para expandir o conhecimento sobre este assunto, bem como para incentivar a criação de programas de auxílio aos estudantes, com o intento de melhorar a aprendizagem daqueles que apresentam déficit na saúde mental.

É apontada pelos autores analisados a relevância da investigação sobre a síndrome de Burnout e o nível de atividade física dos estudantes universitários, pois os indicativos na literatura apontam que baixo nível de atividade física e maus hábitos de saúde, com elevado índices da síndrome de Burnout, são promotores de ações desgastantes no contexto formativo. Da mesma forma, a indicação de estudos de intervenção, considerando a atividade física de estudantes universitários e a síndrome de Burnout, devem ser realizados no interesse de observar a atividade física e a qualidade de vida dos alunos, no intuito de que os estudos estejam centrados em atividades extracurriculares para a prevenção da Síndrome.

\section{Considerações Finais}

$\mathrm{Na}$ literatura sobre a síndrome de Burnout e sobre a atividade física em estudantes universitários consultadas separadamente é perceptível uma gama de estudos que retratam essas temáticas. Por outro lado, é observado que estudos científicos que abordam a temática que 
envolve a relação entre atividade física e Burnout em estudantes universitários são escassos, tendo em vista o reduzido número de investigações que atendiam o objetivo do estudo.

Cabe destacar a fragilidade de investigações no cenário nacional considerando os componentes investigados neste estudo. Todavia, respeitando os procedimentos metodológicos adotados em cada uma das etapas de coleta e análise dos dados, foi manifestada a preocupação em sistematizar estudos sobre o tema em distintas universidades e instituições de ensino superior brasileiras. Além disso, considerando que o contexto internacional demanda um contingente mais amplo de investigações, é lamentável a escassez de estudos encontrados.

De fato, é possível concluir com os estudos encontrados que atividade física e a síndrome de Burnout torna-se significativa por permitir a reflexão e auxiliar na qualidade de ensino, bem como permitir que as universidades viabilizem na sua grade curricular, ou por meio de estratégias extracurriculares, a inserção de atividades físicas no cenário formativo dos estudantes. No cenário universitário, as demandas formativas podem se tornar mecanismos que favoreçam a síndrome de Burnout nos estudantes.

Investigações sobre a temática deste estudo são fundamentais para a efetivação das propostas curriculares e para a elaboração de projetos pedagógicos que visem minimizar os desconfortos ocasionados no período da formação inicial e que busquem a obtenção de melhor qualidade de vida e melhor rendimento acadêmico.

Por fim, na área da Educação Física, percebe-se uma lacuna nas investigações que envolvem a temática atividade física e síndrome de Burnout, evidenciando a necessidade de ampliar as investigações, de modo a contribuir para a produção do conhecimento, bem como incentivar a criação de programas de auxílio aos estudantes, com o intento de garantir a aprendizagem daqueles que apresentam déficit emocional.

\section{Referências}

ALMEIDA, L. S. Transição, adaptação académica êxito escolar no ensino superior. Revista Galego-Portuguesa de Psicoloxía e Educación, Coruna, v. 15, n. 11, p. 1138-1663, 2007.

ARINO, A. P.; HERNANDEZ, J. G. Actividad física y niveles de burnout em alumnos de la E.S.O. Retos-Nuevas Tendencias Em Educacion Fisica Deporte Y Recreacion, Murcia, v. 29, n. 1, p. 95-99, 2016.

BACKOVIĆ, D. V.; ZIVOJINOVIĆ, J. I.;MAKSIMOVIĆ, J.; MAKSIMOVIĆ, M.Gender differences in academic stress and burnout among medical students in final years of education.Psychiatria Danubina, Zagreb, v. 24, n. 2, 2012.

BATISTA, W. S.; ORNELLAS, F. H. Exercício Físico e Depressão: Relação entre o exercício físico e o grau dee depressão. Revista Brasileira de Prescrição e Fisiologia do Exercício, São Paulo, v.7, n. 42, p.474-482, nov./dez. 2013.

BENEVIDES-PEREIRA, A. M. T. Burnout: o processo de adoecer pelo trabalho. In: Benevides, P. A. M. T. (Org.). Burnout: quando o trabalho ameaça o bem-estar do trabalhador. São Paulo: Editora Casa do Psicólogo, 2002. p.21-91. (v. 9).

BIELEMANN, R.; KARINI, G.; AZEVEDO, M. R.; REICHERT, F. F. Prática de atividade física no lazer entre acadêmicos de educação física e fatores associados. Revista Brasileira de Atividade Física e Saúde, Pelotas, v. 12, n. 3, 2007. 
BOING, L.; GUIMARÃES, A. C. A.; REIS, N. M.; RIBOVSK, M. Atividade física após o diagnóstico do câncer de mama: Revisão sistemática. Motricidade, Porto, v. 12, n. 2, p. 155166, 2016.

CABALLERO, C. C.; BRESO, É.; GONZALEZ GUTIERREZ, O. Burnout em estudiantes universitarios. Psicología desde el Caribe, Barranquilla, v. 32, n. 3, p. 424-441, set./dez. 2015.

CAMPBELL J.; PROCHAZKA, A.V.; YAMASHITA, T.; GOPAL, R. Predictors of persistent burnout in internal medicine residents: a prospective cohort study. Academic Medicine, Philadelphia, v. 85, n.10, p. 1630-1634, out. 2010.

CAMPOS, J. A. D. B.; JORDANI, P. C.;ZUCOLOTO, M. L.; BONAFÉ, F. S. S.; MAROCO, J. Síndrome de Burnout tem graduandos de Odontologia. Revista Brasileira de Epidemiologia, São Paulo, v. 15, n. 1, p. 155-65, 2012.

CARLOTTO, M. S.; NAKAMURA, A. P.; CÂMARA, S. G. Síndrome de burnout em estudantes universitários da área da saúde. Psico, Porto Alegre, v. 37, n. 1, p. 57-62, jan./abr. 2006.

CARLOTTO, M. S.; CÂMARA, S. G. Preditores da Síndrome de Burnout em estudantes universitários. Pensamiento Psicológico, Cali, v. 4, n. 10, p. 101-109, 2008.

CARLOTTO, M. S.; CÂMARA, S. G.; OTTO, F.; KAUFFMANN, P. Síndrome de Burnout e coping em estudantes de Psicologia. Boletim de Psicologia, São Leopoldo, v. 59, n. 131, p. 167-178, dez. 2010.

CECIL, J.; CALUM, M. H.; HART, J.; LAIDLAW, A. Behaviour and burnout in medical students. Medical Education Online, Hoboken, v. 19, ago. 2014.

DOWNS, S. H.; BLACK, N. The feasibility of creating a checklist for the assessment of the methodological quality both of randomized and nonrandomized studies of health care interventions. Journal of Epidemiology and Community Health, Indiana, v. 52, n. 52, p. 377384, jun. 1998.

DYRBYE, L. T.; SHANAFEL, T. A narrative review on burnout experienced by medical students and residents. BMC Medical Educacion, London, v. 50, n.1, p. 132-149, jan. 2016.

FARES, J.; SAADEDDIN, Z.; TABOSH, H.; ARIDI, H.; MOUHAYYAR, C.; KOLEILAT, M. K.; CHAAYA, M.; ASMAR, K. Extracurricular activities associated with stress and burnout in preclinical medical students. Journal of Epidemiology Global Health, Amsterdam, v. 6, n. 3, p. 177-185, set. 2015.

FLESHNER, M. Physical activity and stress resistance: sympathetic nervous system adaptations prevent stress induced immunosuppression. Exercise and Sport Sciences Reviews, Hagerstown, v. 33, p. 120-126, jul. 2005.

FOGAÇA, M.C.; MATOS, D. C.; BORSETTI, J. S.; DI RIENZO, V. D.; RIBEIRO, L. P.; ZIMBARDI, R.; SILVA, I. G. Academic experience of Psychology students: Differences be- 
tween beginners and graduating. Estudos de Psicologia, Campinas, v. 33, n. 3, p. 515-523, jul./set. 2016.

FONTES, A.C. D.; VIANNA, R.P.T. Prevalência e fatores associados ao baixo nível de atividade física entre estudantes universitários de uma universidade pública da região Nordeste Brasil. Revista Brasileira de Epidemiologia, São Paulo, v. 2, n.1, p. 20-29, 2009.

FREITAS, A. R.; CAMESECA, E. C.; PAIVA, C. E.; PAIVA, B. S. R. Impacto de um programa de atividade física sobre a ansiedade, depressão, estresse ocupacional e síndrome de Burnout dos profissionais de enfermagem no trabalho. Revista Latino-americana de Enfermagem, São Paulo, v. 22, n. 2, p. 332-336, mar./abr. 2014.

GALDINO, M. J. Q.; MARTINS, J. T.; HADDAD, M. C. F. L.; ROBAZZI, M. L. C. C.; BIROLIM, M. M. Síndrome de Burnout entre mestrandos e doutorandos em enfermagem. Acta Paulista de Enfermagem, São Paulo, v. 29, n. 1, p. 100-106, 2016.

GARBIN, S. C. A; SALIBA, N. A.; SANTOS, R. R, PRADO, R.; GARBIN, A. J. Burnout em estudiantes de odontología: evaluacion a través mbi - version estudiantes. Medicina $\mathbf{y}$ seguridade del trabajo, Madrid, v. 58, n. 229, p. 327-334, out./dez. 2012.

GÓMEZ, H. P.; PÉREZ, C. V.; PARRA, P. P.; ORTIZ, L. M.; MATUS, O. B.; MCCOLL, P. C.; TORRES, G. A.; ANDREA MEYER, A. K. Relación entre el bienestar y el rendimiento académico em alumnos de primer año de medicina. Revista de Medicina do Chile, Santiago, v. 143, p. 930-937, 2015.

GUEDES, D. P.; GUEDES, J. E. R. P. Exercício Físico na promoção da saúde. Londrina: Midiograf, 1995.

GUIMARÃES, E. R. A Síndrome de Burnout em estudantes de ciências contábeis: pesquisa na cidade de São Paulo. 2014. Dissertação (Mestrado em Ciências Contábeis) - Fundação Escola de comércio Álvaro Penteado, São Paulo, 2014.

LEITÃO, M. B.; LAZZOLI, J. K.; OLIVEIRA, M. A. B.; NÓBREGA, A. C. L. Posicionamento Oficial da Sociedade Brasileira de Medicina do Esporte: Atividade Física e Saúde na Mulher. Revista Brasileira de Medicina e Esporte, São Paulo, v. 6, n. 6, nov./dez. 2000.

MANOSSO, M.; LANFERDINI, F. J.; DAL'AGNOL, M. J.; RONCADA, C.; DIAS, C. P. Comparação dos níveis de estresse e estilo de vida entre praticantes e não praticantes de ginástica laboral. Revista Brasileira de Ciências e movimento, Brasília, v. 22, n. 2, p. 65-71, 2014.

MARCONDELLI, P.; COSTA, T. H. M.; SCHMITZ, B. A. S. Nível de atividade física e hábitos alimentares de universitários do $3^{\circ}$ ao $5^{\circ}$ semestres da área da saúde. Revista de Nutrição, Campinas, v. 21, n. 1, p. 39-47, jan./fev. 2008.

MASLACH, C.; JACKSON, S. E. Maslach Burnout Inventory. Palo Alto: Consulting Psychologists Press, 1981. 
MATSUDO, S. M.; MATSUDO, V. K. R. Evidencias da importância da atividade física nas doenças cardiovasculares e na saúde. Revista Diagnóstico e tratamento, São Paulo, v. 5, n. 2, 10-17, jul. 2000.

MEJÍA, F. M. Efectos de ladanza terapêutica em el control del estrés laboral em adultos entre 25 y 50 años. HacialaPromoción de laSalud, Manizales, v. 16, n. 1, p. 156-174, jan./mar. 2011.

MELLO, M. T.; BOSCOLO, R.A; ESTEVES, A. M.; TUFIK, S. Exercício Físico e os Aspectos Psicobiológicos. Revista Brasileira de Medicina e Esporte, São Paulo, v. 11, n. 3, maio/jun. 2005.

MESSAS, J. T.; LEONELlO, V. M.; FERNANDES, M. F. P.; GONÇALVES, G. C. C.; BUCCHI, S. M.; MIRA, V. L. O ambiente educacional do curso de Graduação em Enfermagem na perspectiva dos estudantes. Revista da Escola de Enfermagem da USP, São Paulo, v. 49, n. 2, p. 106-114, 2015.

MOHER, D.; LIBERATI, A.; TETZLAFF, J.; ALTMAN, D.G. The PRISMA Group Preferred Reporting Items for Systematic Reviews and Meta-Analyses: The PRISMA Statement. PloS Medicine, Califórnia, v.6, n. 6, jul. 2009.

MORI, M. O.; VALENTE T. C. O.; NASCIMENTO L. F. C. Síndrome de Burnout e Rendimento Acadêmico em Estudantes da Primeira à Quarta Série de um Curso de Graduação em Medicina. Revista Brasileira de Educação Médica, Rio de Janeiro, v. 36, n. 4, p. 536-540, ago./out. 2012.

MURILLO-PÉREZ, L.; ROJAS-ADRIANZÉN, C.; RAMOS-TORRES, G.; CÁRDENASVICENTE, B.; HERNÁNDEZ-FERNÁNDEZ, W.; LARCO-CASTILLA, P.; GARCÍA, L.; MEZONES-HOLGUÍN, E. Asociación entre el riesgo de depresión mayor y el bajo nivel de actividad física entra bajadores peruanos que cursan estudios universitários. Revista Peruana de la medicina y la salud pública, Lima, v. 31, p. 520-524, fev./jun. 2014.

NAHAS, M.V; BARROS, M. V. G.; FRANCALACCI, V. L. O Pentáculo do bem-estar: base conceitual para avaliação do estilo de vida de indivíduos e grupos. Revista Brasileira de Atividade Física e Saúde, Pelotas, v. 5, n. 2, p. 48-59, 2000.

RODRIGUES, E. S. R; CHEIK, N. C.; MAYER, A. F. Nível de atividade física e tabagismo em universitários. Revista de Saúde Pública, São Paulo, v. 42, n. 4, p. 672-678, ago. 2008.

RONCADA, C.; DIAS, C. P. Comparação dos níveis de estresse e estilo de vida entre participantes e não participantes de ginástica laboral. Revista Brasileira de Ciência e Movimento, Brasília, v. 22, n. 2, 65-71, mar./abr.2014.

ROSALES; R. Y. Estudio unidimensional del síndrome de Burnout em estudiantes de medicina de Holguín. Revista de la Asociación Española de Neuropsiquiatría, Madrid, v.32, n.116, p. 795-803, out./dez. 2012. 
ROSALES, R. Y.; ROSALES PANEQUE, F. R. Burnout estudiantil universitario: Conceptualización y estudio. SaludMent, Colonia San Lorenzo Huipulco, v. 36, n. 4, p. 337-345, out./dez. 2013.

SALAZAR, F. V. Ejercicio y depresión. Revista Colombiana de Psiquiatria, Bogota, v. 39, n. 4, jul./out. 2010.

SANTOS, T. S.; ANDRADE LEAO. O. M.; LEITE, J. S.; SILVA, M. C. Atividade física em acadêmicos de Educação Física: um estudo longitudinal. Revista Brasileira de Atividade Física e Saúde, Pelotas, v. 22, n. 1, p. 76-84, 2017.

SCHENKEL, I. C.; BÜNDCHEN, D. C.; QUITES, M. P.; SANTOS, R. Z.; CARVALHO, T. Comportamento da Pressão Arterial em Hipertensos após Única Sessão de Caminhada e de Dança de Salão: estudo preliminar. Revista Brasileira de Cardiologia, Rio de janeiro, v. 24, n. 1, p. 26-32, jan./fev. 2011.

SILVA, M. C.; OLIVERIA, J. M. S.; ALVES, L.; LEPSEN, A. M. Atividade física e fatores associados em doentes arteriais coronarianos. Scientia Medica, Rio Grande do Sul, v.24, n. 1, p. 46-53, 2014.

SILVA, P. A. C.; SIMÕES, V. P.; VAZ, M. C. S. Nível de atividade física no lazer e deslocamento e fatores associados em alunos de educação física em Coimbra - Portugual. Revista Brasileira de Atividade Física e Saúde, Pelotas, v. 20, n. 6, p. 559-568, nov. 2015.

SOUZA, R. S.; TRIGUEIRO, R. P. C.; ALMEIDA, T. N. V.; OLIVEIRA, J. A. A pósgraduação e a Síndrome de Burnout: estudo com alunos de Mestrado em Administração. Revista Pensamento Contemporâneo em Administração, Rio de Janeiro, v. 4, n. 3, p. 12-21, set./dez. 2010.

SZILVIA, Á.; ANIKÓ, N.; KATALIN, N.; ANIKÓ, H. A megküzdési stratégiák negatív és pozitív prediktív kapcsolata a kiégés három dimenziójával orvostanhallgatók körében. OrvosiHetilap, Budapest, v. 155, n. 32. p. 1273-1280, 2014.

TOMASCHEWSKI-BARLEM, J. G.; LUNARDI, V. L.; RAMOS, A. M.; SILVEIRA, SILVA, R.; DEVOS BARLEM, EDISON LUIZ; ERNANDES, CAROLINA MIRAPALHETA. Manifestações da Síndrome de Burnout entre estudantes de graduação em enfermagem. Texto Contexto Enfermagem, Florianópolis, v. 22, n. 3, p. 754-762, jul./set. 2013.

WEIGHT, C. J.; SELLON， J. L.; LESSARD-ANDERSON， C. R.; SHANAFELT, T. D.; OLSEN, K. D.; LASKOWSKI, E. R. Physical activity, quality of life, and burnout among physician trainees: the effect of a team-based, incentivized exercise program. Mayo Clinic Proceedings, Amsterdam, v. 88, n.12, p. 1435-1442, dez. 2013.

Recebido em: 28/03/2018

Revisado em: 13/07/2018

Aprovado em: 10/08/2018 
Endereço para correspondência:

fariasgel@hotmail.com

Gelcemar Oliveira Farias

Universidade do Estado de Santa Catarina, Florianópolis, Santa Catarina, Brasil.

Centro de Educação Física e Desportos.

Rua Pascoal Simone

Coqueiros

88080350 - Florianópolis, SC - Brasil 